\title{
Influence of atrial ultrastructural remodeling on its early mechanical transport following surgery for atrial fibrillation and mitral insufficiency
}

\author{
T Kopjar $^{1 *}$, H Gasparovic ${ }^{1}$, M Cikes², V Velagic ${ }^{2}$, Z Colak ${ }^{3}$, LJ Hlupic ${ }^{4}$, M Petricevic ${ }^{1}$, L Svetina ${ }^{1}$, T Fabijanić 1 , \\ D Milicic ${ }^{2}$, B Biocina $^{1}$
}

From 23rd World Congress of the World Society of Cardio-Thoracic Surgeons

Split, Croatia. 12-15 September 2013

\section{Background}

Establishment of sinus rhythm (SR) following radiofrequency ablation (RFA) for longstanding persistent atrial fibrillation (AF) is not necessarily an equivalent to physiological atrial mechanical activity. We aimed to determine the influence of atrial ultrastructural remodeling on the recovery of its mechanical transport following restitution of SR.

\section{Methods}

We enrolled 32 patients operated for severe mitral regurgitation. Half of the patients had no history of AF constituting the SR group. The remaining half had concomitant RFA for longstanding persistent AF, the RFA group. Intraoperative transesophageal echocardiography was used for tissue Doppler indices (TDI) data acquisition at the left atrial (LA) lateral wall. During post-procedural data acquisition all patients were in SR. LA biopsies were obtained during surgery and quantified for fibrosis after Mallory's trichrome staining.

\section{Results}

Atrial mechanical contraction was noted in both groups following surgery, although TDI values of late diastolic strain rate (SRI A') and tissue velocity (TVI A') were superior in the SR group $(-1.49 \pm 1.04$ vs. $-2.82 \pm 1.97$, $\mathrm{p}=0.022 ; 1.22 \pm 1.08$ vs. $3.86 \pm 2.30, \mathrm{p}=0.003)$. Postprocedural atrial filling was compromised in the RFA (TVI S' $-4.20 \pm 2.27$ vs. $-2.70 \pm 1.13, \mathrm{p}=0.031$ ) and the SR group (SRI S' $4.31 \pm 3.03$ vs. $3.93 \pm 2.22, \mathrm{p}=0.009$ ).
Negative correlation between fibrosis and the preprocedural SRI A' was noted in the SR group $(r=-0.69$, $\mathrm{p}=0.015)$, although not significant throughout both groups $(\mathrm{r}=-0.33, \mathrm{p}=0.088)$.

\section{Conclusions}

We have shown that the restoration of SR following RFA and mitral valve surgery is not equal to normal atrial mechanical transport. Interstitial fibrosis, a symbol of ultrastructural remodeling, is a negative precursor of LA mechanical activity. Our data did not corroborate clear difference in the extent of fibrosis between groups, although a tendency towards more severe ultrastructural remodeling was noticed in patients with history of AF.

\section{Authors' details}

'Department of Cardiac Surgery, University Hospital Centre Zagreb, Zagreb, Croatia. ${ }^{2}$ Department of Cardiology, University Hospital Centre Zagreb, Zagreb, Croatia. ${ }^{3}$ Department of Anesthesiology, University Hospital Centre Zagreb, Zagreb, Croatia. ${ }^{4}$ Department of Pathology, University Hospital Centre Zagreb, Zagreb, Croatia.

Published: 11 September 2013

doi:10.1186/1749-8090-8-S1-063

Cite this article as: Kopjar et al:: Influence of atrial ultrastructural

remodeling on its early mechanical transport following surgery for atrial fibrillation and mitral insufficiency. Journal of Cardiothoracic Surgery 2013 8(Suppl 1):063.

\footnotetext{
* Correspondence: tkopjar@gmail.com

'Department of Cardiac Surgery, University Hospital Centre Zagreb, Zagreb, Croatia

Full list of author information is available at the end of the article
}

(c) 2013 Kopjar et al; licensee BioMed Central Ltd. This is an Open Access article distributed under the terms of the Creative Commons 